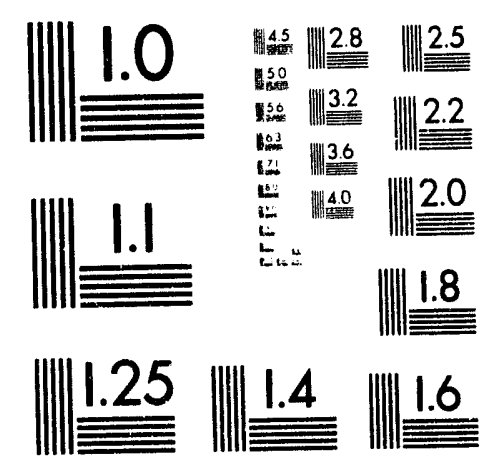



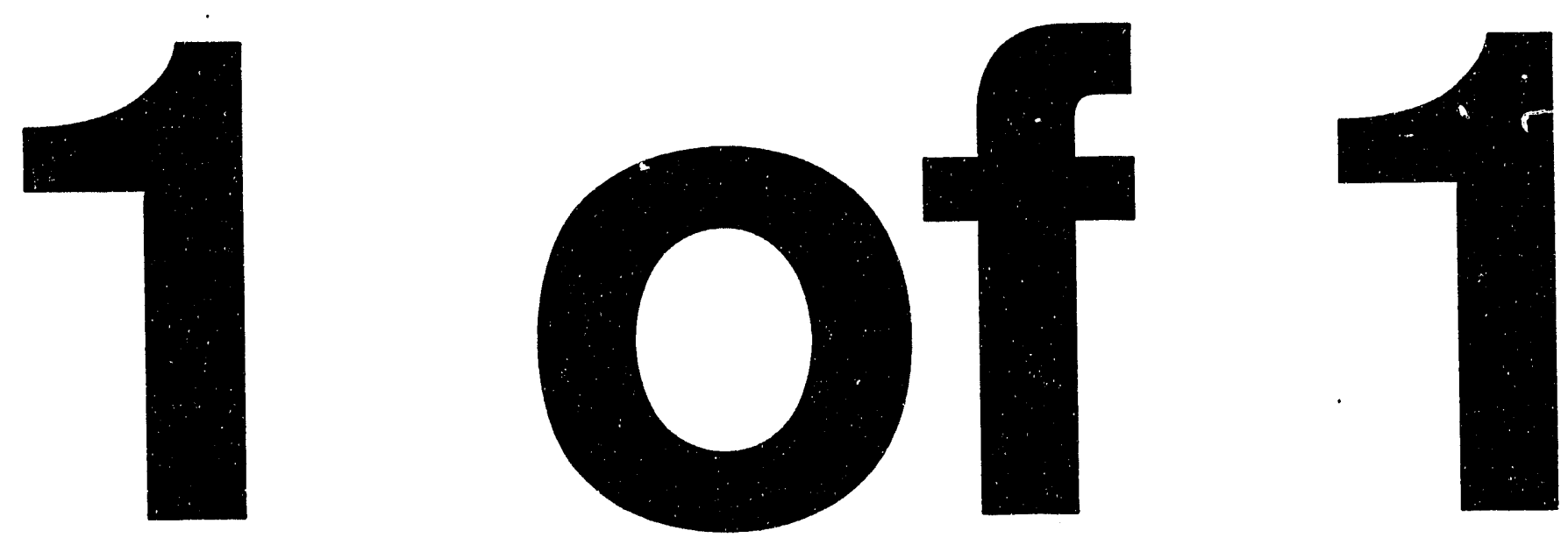
LBL-33309

\title{
Emittance Measurement and Modeling of the ALS 50 MeV Linac to Booster Line
}

\author{
J. Bengtsson, W. Leemans, and $\Gamma$. Byrne \\ Accelerator and Fusion Research Division \\ Lawrence Berkeley Laboratory \\ University of California \\ Berkeley, California 94720
}

May 1993

This work was supported bj the Director, Office of Energy Research, Office of High Nuclear Physics, High Energy Physics Division, of he U.S. Department of Energy under Contract No. DE-AC03-76SF00098.

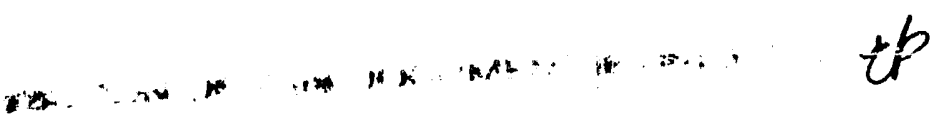




\title{
Emittance Measurement and Modeling of the ALS $50 \mathrm{MeV}$ Linac to Booster Line *
}

\author{
J. Bengtsson, W. Leemans and T. Byrne \\ Lawrence Berkeley Laboratory, University of California \\ 1 Cyclotron Road, Berkeley, CA 94720, USA
}

\section{Abstract}

The Twiss parameters and emittance have been measured for the ALS LINAC through non-linear $\chi^{2}$ fitting of the measured beam size (fixed location) vs. focal strength of a quadrupole triplet. The obtained values have been used to calculate the expected beam size and dispersion along the linac-to-booster transport line giving good agreement with measurements. The efforts resulted in superior injection into the booster.

\section{INTRODUCTION}

The Advanced Light Source at LBL has a $50 \mathrm{MeV}$ linac, a booster which increases the electron energy to $1.5 \mathrm{GeV}$ and a storage ring which is expected to need refilling every $6-8$ hours. In between refills the $50 \mathrm{MeV}$ electron will be transported into an experimental vault and will be used to conduct a number of experiments such as plasma lens focusing, generation of femtosecond $\mathrm{X}$-ray pulses and interaction of the electron beam with a variety of electromagnetic cavities [1].

To be able to design a beam transport line optimized for the different experiments, we have measured the beam's initial conditions after the linac. In addition, these values have also been used to calculate the beam size and dispersion along the Linac-To-Booster (LTB) transport line and compared with measurements.

\section{THEORETICAL MODEL}

\section{A. Equations of motion} by [2]

The linearized transverse motion of the beam is described

$$
x(s)=\sqrt{\varepsilon_{x} \beta(s)} \cos \left[\mu_{x}(s)+\varphi_{x}\right]+\eta_{x}(s) \delta
$$

using the phase space coordinates $\bar{x}=\left(x, p_{x}, y, p_{y}, \delta\right)$, where $\beta$ is the beta-function, $\mu$ the phase advance, $\eta$ the dispersion and $\varepsilon$ a constant of motion known as the emittance. The beam is parameterized by the six parameters $\alpha_{x}, \beta_{x}, \varepsilon_{x}, \alpha_{y}, \beta_{y}$ and $\varepsilon_{y}$ at each point along the transport line. Since we have three parameters in each plane but can only observe configuration space, i.e. beam sizes, we need at least three different observations of beam size in each plane to be able to determine the corresponding beam parameters at a given point along the transport line.

\section{B. Statistical Analysis by Non-linear $\chi^{2}$}

\footnotetext{
*This work was supported by the Director, Office of Energy Research, Office of B asic Energy Sciences, Materials Sciences Division. of the U.S. Department of Energy under Contract No. DEAC03-76SF00098.
}

The horizontal beam size $x$ is a function of $\alpha_{x}, \beta_{x}$ and $\varepsilon_{x}$

$$
x(\overline{\mathrm{q}} \overline{\mathrm{a}})=\sqrt{\varepsilon_{x}\left[\mathrm{~m}_{11}^{2} \beta_{x}(\overline{\mathrm{q}})-2 \mathrm{~m}_{11} \mathrm{~m}_{12} \alpha_{x}(\overline{\mathrm{q}})+\mathrm{m}_{12}^{2} \frac{1+\alpha_{x}^{2}(\overline{\mathrm{q}})}{\beta_{x}(\overline{\mathrm{q}})}\right]}
$$

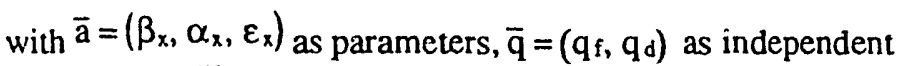
variables and $m_{\mathrm{ij}}$ being the elements of the transport matrix $\overline{\mathrm{x}}_{\mathrm{f}}=\mathrm{M} \overline{\mathrm{x}}_{\mathrm{i}}$. The $\chi^{2}$ merit function is

$$
\chi^{2}(\bar{a})=\sum_{i=1}^{N}\left[\frac{x_{i}-x\left(\bar{q}_{i ;} ; \bar{a}\right)}{\sigma_{i}}\right]^{2}
$$

where $\mathrm{N}$ is the number of data points, i.e. measured and computed beam size. $\chi^{2}$ is minimized by solving the system

$$
\begin{aligned}
& \sum_{i=1}^{N} u_{k} \delta a=u_{k}, \quad u_{k} \equiv-\frac{1}{2} \frac{\partial \chi^{2}}{\partial a_{k}}=\sum_{i=1}^{N} \frac{\left[x_{i}-x\left(\bar{q}_{i} ; \bar{a}\right)\right]}{\sigma_{i}^{2}} \frac{\partial x\left(\bar{q}_{i} ; \bar{a}\right)}{\partial a_{k}} \\
& v_{d d} \equiv \frac{1}{2} \frac{\partial^{2} \chi^{2}}{\partial a_{k} \partial a}=\sum_{i=1}^{N} \frac{1}{\sigma_{i}^{2}} \frac{\partial x\left(\bar{q}_{i} ; \bar{a}\right)}{\partial a_{k}} \frac{\partial x\left(\bar{q}_{i j} ; \bar{a}\right)}{\partial a}+O\left(\partial^{2}\right)
\end{aligned}
$$

and iterating [3]. The second order derivatives have been neglected to stabilize the iterations. We find

$$
\begin{aligned}
u_{1}= & -\frac{1}{2} \frac{\partial \chi^{2}}{\partial \beta_{x}}=\sum_{i=1}^{N} \frac{\left[x_{i}-x\left(\bar{q}_{i} ; \bar{a}\right)\right]}{\sigma_{i}^{2}} \frac{\varepsilon_{x}\left(m_{11}^{2}-m_{12}^{2} \frac{1+\alpha\left(\bar{q}_{i}\right)}{\beta_{x}^{2}\left(\bar{q}_{i}\right)}\right)}{2 \sqrt{ }}, \\
u_{2}= & -\frac{1}{2} \frac{\partial \chi^{2}}{\partial \alpha_{x}}=\sum_{i=1}^{N} \frac{\left[x_{i}-x\left(\bar{q}_{i} ; \bar{a}\right)\right]}{\sigma_{i}^{2}} \frac{\varepsilon_{x}\left(-m_{11} m_{12}+m_{12}^{2} \frac{\alpha_{x}\left(\bar{q}_{i}\right)}{\beta_{x}\left(\bar{q}_{i}\right)}\right)}{\sqrt{ }}, \\
u_{3}= & -\frac{1}{2} \frac{\partial \chi^{2}}{\partial \varepsilon_{x}}=\sum_{i=1}^{N} \frac{\left[x_{i}-x\left(\bar{q}_{i} ; \bar{a}\right)\right]}{\sigma_{i}^{2}} \\
& \times \frac{m_{11}^{2} \beta_{x}\left(\bar{q}_{i}\right)-\angle m_{11} m_{12} \alpha_{x}\left(\bar{q}_{i}\right)+m_{12}^{2} \frac{1+\alpha_{x}^{2}\left(\bar{q}_{i}\right)}{\beta_{x}\left(\bar{q}_{i}\right)}}{2 \sqrt{ }}
\end{aligned}
$$

where

$$
\sqrt{ } \equiv \sqrt{\left.\varepsilon_{x}\left(m_{11}^{2} \beta \bar{q}_{i}\right)-2 m_{11} m_{12} \alpha_{x}\left(\bar{q}_{i}\right)+m_{12}^{2} \frac{1+\alpha_{x}^{2}\left(\bar{q}_{i}\right)}{\beta_{x}\left(\bar{q}_{i}\right)}\right)}
$$

The confidence interval is given by

$$
\delta a_{i}= \pm \sqrt{\Delta \chi^{2}(v)^{-1}}
$$

where we choose $\Delta \chi^{2}=4$ for a $95.4 \%$ confidence interval. 
where we choose $\Delta \chi^{2}=4$ for a $\$ 5.4 \%$ confidence interval.

\section{EMITTANCE MEASUREMENT}

\section{A. Linac parameiers}

The parameters of the linse are given in Table 1 . The LTB line transports the beam tow'ards the $1.5 \mathrm{GeV}$ booster and must be tuned to provide the correct matching conditions into the ring.

$\begin{array}{ll}\text { Maximum Energy } & 50 \mathrm{MeV} \\ \text { Charge } & 1-2 \mathrm{nC} / \text { bunch } \\ \text { Bunch Length }\left(\sigma_{\mathrm{Z}}\right) & 10-15 \mathrm{ps} \\ \text { Emittance rms (unnorm) } & 0.3 \mathrm{~mm} \text {-mrad } \\ \text { \# bunches/macro pulse } & 1-10(\max 100) \\ \text { @ } 125 \mathrm{MHz} & \\ \text { Macro pulse rep. rate } & 1-10 \mathrm{~Hz}\end{array}$

Table 1: ALS Linac parameters

Typically, emittance measurements are done with a pepper pot and beam profiles are measured with wire scans or harps. We have carried out the measurements by measuring the beam size on a fluorescent screen as a function of the focal strength of the focusing element, $Q_{12}$, of a quadrupole triplet, while keeping the detocusing element, $Q_{11}$, at a fixed strength.

The beam size is obtained as follows: light emitted by the fluorescent screen, is imaged onto a CCD-camera. The video signal output is frame-grabbed with 8 bit resolution on a Macintosh computer and analyzed using commercial image processing software. For the particular choice of magnification, the intrinsic spatial resolution of the system is about $110 \mu \mathrm{m}$ but the statistical analysis, discussed previously, improves this about five-fold to $20 \mu \mathrm{m}$. For fixed imaging conditions, we have evaluated the linearity of the system by measuring the peak number of counts on the image as a function of number of bunches per macro pulse. The complete imaging system was found to be linear, for beam intensities below those resulting in 200 counts on the image. The noise floor is typically 15 counts.

After acquisition of an image, horizontal and vertical lineouts were fit to a Gaussian distribution using a non-linear Levenberg Marquardt fitting routine [3] with 3 parameters: noise floor (counts), amplitude (counts) and $\mathrm{ms}$ beam size.

\section{Emittance measurement.}

The measurements were carried out as follows. First, all quadrupole magnets were cycled. At the end of the cycling procedure the current was brought up to $90 \%$ of the final setvalue and then slowly increased to the $100 \%$ value. Without these procedures, preliminary measurements indicated that hysteresis effects led to irreproducible results. For a given current setting of the focusing (defocusing) element, the current delivered to the defocusing (focusing) element was varied from 0 to $8 \mathrm{~A}$ (maximum current) in steps of $0.5 \mathrm{~A}$. An example of the horizontal and vertical beam sizes as a function of current is shown in Fig. 1.

The experimental results were modeled with TRACY [4] using the measured location of the different beam line components (dipole and quadrupole magnets, fluorescent screens); magnetic lengths and stren.gths. The magnetic length for each quadrupole was calculated from the measured B'Leff line integral value and the calculated field gradient, for a given current setting. All the current supplies were carefully calibrated. The measured transfer functions of the quadrupoles were used to calculate the focusing strength .

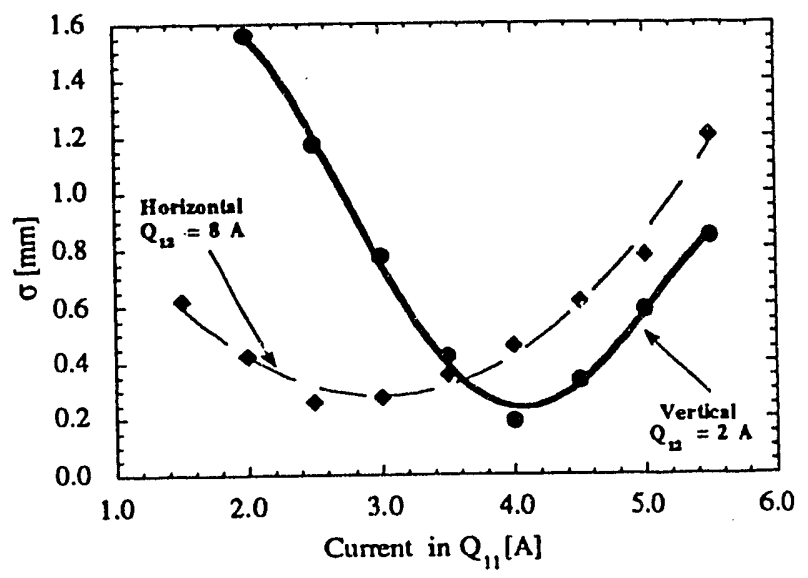

Figure 1: Horizontal and vertical beam sizes as a function of current in $Q_{11}$ for fixed current in $Q_{12}$. The lines through the data are polynomial fits used in "he modeling.

The results of the non-linear $\chi^{2}$ from are sun marized in Table 2. '

\begin{tabular}{|l|c|}
\hline$\alpha_{X}$ & $1.31 \pm 0.12$ \\
\hline$\beta_{X}[\mathrm{~m}]$ & $3.09 \pm 0$ \\
\hline$\varepsilon_{X} \mathrm{rms}$, unnorm. [mm-mrad] & $0.32 \pm 0.02$ \\
\hline$\alpha_{y}$ & $-0.19 \pm(1.11$ \\
\hline$\beta_{Y}[\mathrm{~m}]$ & $1.00 \pm 1) .10$ \\
\hline$\varepsilon_{y} \mathrm{rms}$, unnorm. [mm-mrad] & $0.33 \pm 0.03$ \\
\hline
\end{tabular}

Table 2: Measured initial conditions for the $50 \mathrm{MeV}$ beam.

\section{MODELING OF THE LTB-L'NE}

The measured initial conditions allow us to calculate the beam sizes and dispersion along the linac-to-booster (LTB) line and to compare with measured values. Previous operation of the LTB-line utilized quadrupoles set points giving predicted beam sizes shown in Fig. 2. Visual inspection of beam profiles, aided by image enhancing software, indicated beam scraping. Furthermore, one could also notice considerable beam motion at the injection point. This is explained by rather large dispersion at that point and the pulse to pulse energy jitter of the linac.

However, the measured initial conditions allow us to calculate the proper settings of the quadrupole power supplies for matching the beam into the booster. The beam profiles were measured at 5 different locations along the LTB-line for these settings. The comparison between experimental and theoretical results is given in Table 3 . Notice, that a small modeling error of the dispersion at one point will be substantially magnified in downstream quadrupoles. No 
fluorescent screen is present after $B 1$ preventing us from measuring dispersion after the achromat (BS, Q2 and B1).

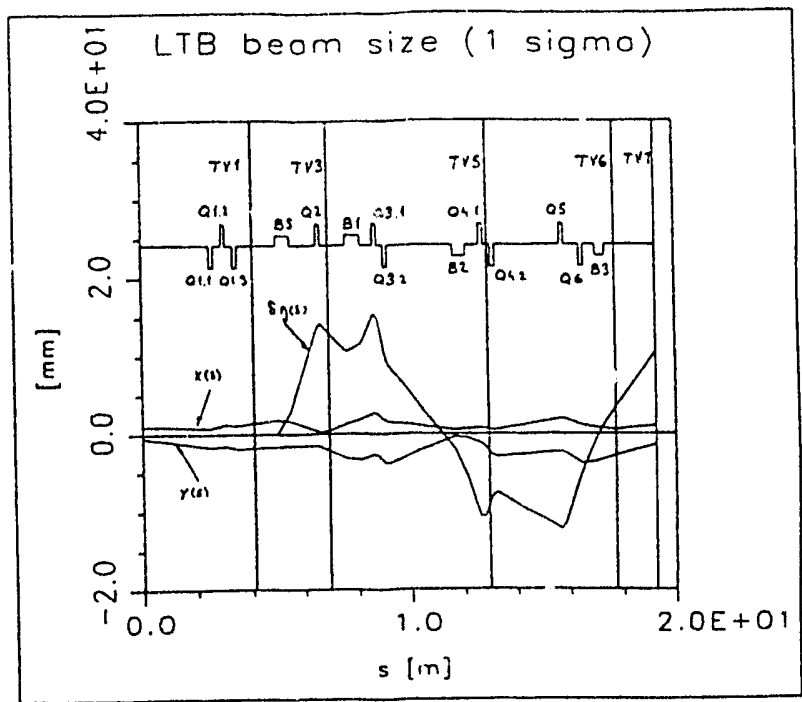

Figure 2: Original predicted beam profile along the LTB line and the effect of dispersion.

The predicted beam profiles for the optimized settings are shown in Fig. 3.

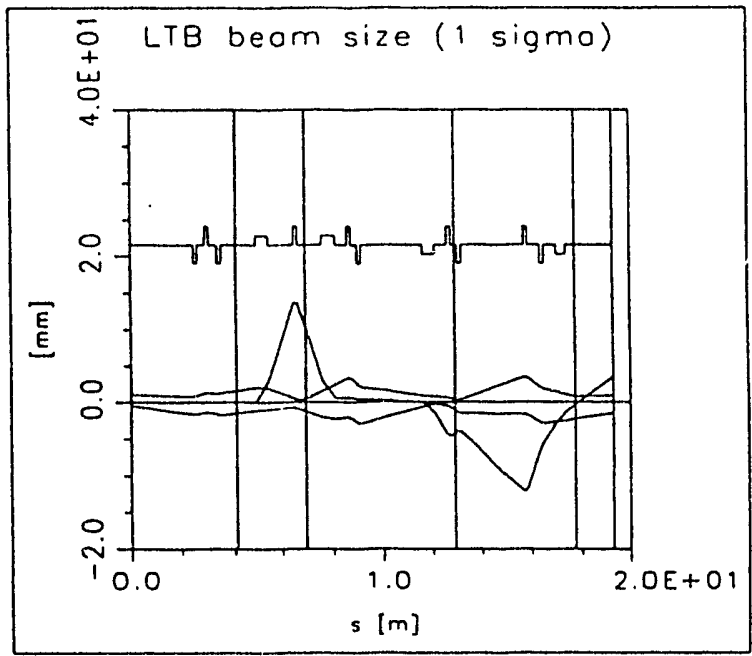

Figure 3: Optimized LTB-line, tuned for minimum dispersion at injection into the booster.

The experimentally measured vertical beam sizes is given by $\sigma=\sqrt{\varepsilon \beta}$. However, analysis of the horizontal beam profiles is complicated by the fact that beam loading of the accelerator structure leads to systematic energy difference between consecutive bunches of $0.8 \%$. This causes on!y partial spatial overlapping of the beam profiles on screens located in dispersive sections. The need for running more than 1 bunch arose from the limited light sensitivity of the imaging system. With the present LINAC performance, adding consecutive images to increase the dynamic range cannot be done: the energy jitter between consecutive macro-pulses was found to be as high as $1 \%$. We are currently working on improving the sensitivity of the imaging system by an order of magnitude.

\begin{tabular}{|c|c|c|c|c|}
\hline Loc. & $\begin{array}{c}\text { Measured } \\
\sigma_{\text {hor }} \\
{[\mathrm{mm}]}\end{array}$ & $\begin{array}{c}\text { Model } \\
\sigma_{\text {hor }} \\
{[\mathrm{mm}]}\end{array}$ & $\begin{array}{c}\text { Mr.asured } \\
\sigma_{\text {ver }} \\
{[\mathrm{mm}]}\end{array}$ & $\begin{array}{c}\text { Model } \\
\sigma_{\mathrm{ver}} \\
{[\mathrm{mm}]}\end{array}$ \\
\hline tv1 & 1.67 & $1.53,1.53$ & 1.76 & 1.52 \\
\hline tv3 & 9.15 & $10.69,0.33$ & 1.16 & 1.28 \\
\hline tv4 & 2.88 & $4.69,0.37$ & 0.99 & 1.06 \\
\hline tv5 & 2.49 & $1.08,0.70$ & 1.76 & 2.36 \\
\hline tv6 & 0.81 & $4.30,0.88$ & 1.30 & 1.52 \\
\hline
\end{tabular}

Table 3: Experimental and theoretical beam sizes along the LTB-line. The first and second entry in the third column are with and without dispersion taking into account, respectively.

To obtain the linear dispersion, the change of beam location $\Delta x$ on TV3 ( 22 off) was measured as a function of the strength of the upstream BS dipole magnet. By using

$$
\begin{aligned}
\Delta x & =\delta \eta_{x}=\delta\left[\rho_{0}(1-\cos \phi)+L_{\text {drift }} \sin \phi\right], \\
\delta & =-\Delta B_{\perp} \frac{\rho_{0}}{\left(B_{\perp} \rho_{0}\right.}+O(2)
\end{aligned}
$$

we find a measured dispersion $\eta_{\mathrm{x}}=1.18 \mathrm{~m}$ which agrees well with the predicted value of $1.13 \mathrm{~m}$.

\section{CONCLUSIONS}

A detailed quantitative analysis has been presented on a) the measurement of the beam emittance and Twiss parameters of the ALS Linac, and b) on the modeling and optimization of the LTB line. The solution for the beam's initial conditions was propagated down the LTB-line. Good agreement between the calculated and measured beam sizes and dispersion was obtained. Furthermore, the quantitative and qualitative analysis of beam profiles helped us to diagnose incorrect operation of the Linac sub-harmonic bunching system, calibration problems with power supplies and.the importance of magnet cycling to avoid systematic errors caused by hysteresis effects. Finally, it has led to a substantial improvement of injection stability of the beam into the booster.

\section{ACKNOWLEDGMENT}

The authors would like to thank S. Chattopadhyay and the Center for Beam Physics for continuos support and encouragement, and the ALS operations team for their cooperation.

\section{REFERENCES}

[1] W. Leemans et al., these proceedings.

[2] E. D. Courant and H. S. Snyder, Ann. Phys. Vol 3, p. 1 (1958).

[3] W. H. Press, B. P. Flannery, S. A. Teukolsky and W. T. Vetterling, "Numerical Recipes" (Cambridge University Press) 1989.

[4] J. Bengtsson, E. Forest and H. Nishimura, unpublished. 

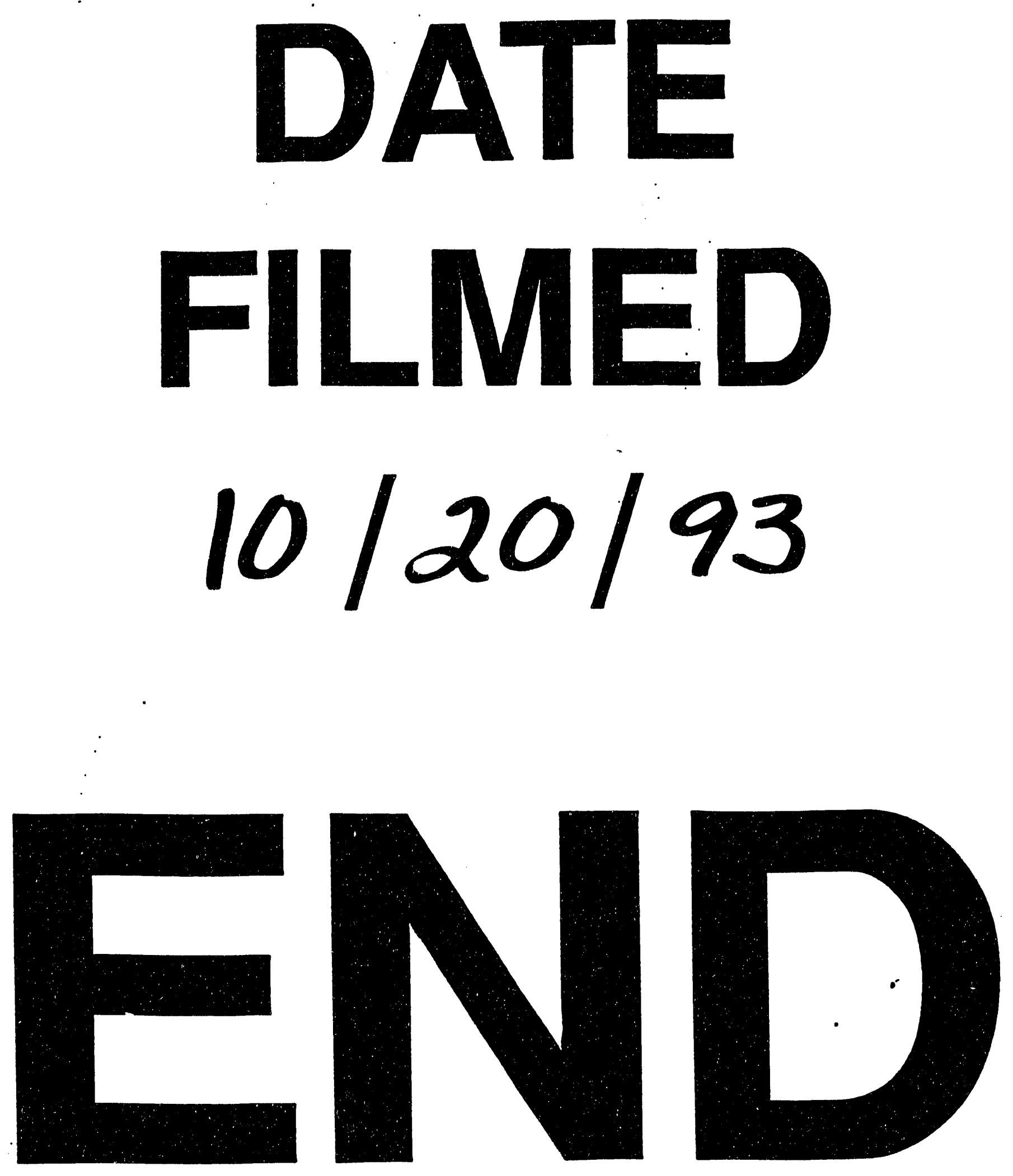
